\title{
WEIGHT SPACES OF LIE ALGEBRA MODULES
}

\author{
MARTHA K. SMITH ${ }^{1}$
}

\begin{abstract}
Let $V$ be a finite-dimensional module for the finite-dimensional Lie algebra $L$ over a field of characteristic zero. If $V^{\lambda}=\{v \in V \mid$ all $x \in$ $L,[x-\lambda(x)]^{i} v=0$ for some $\left.i\right\}$ is nonzero, then $\lambda \in L^{*}$ and is a character of $L$. Moreover, the corresponding eigenspace $\{v \in V \mid$ all $x \in L, x v=\lambda(x) v\}$ is nonzero and $V^{\lambda}$ is an $L$ submodule of $V$.
\end{abstract}

Let $L$ be a finite-dimensional Lie algebra over the field $k, V$ a finite-dimensional $L$ module. If $\lambda$ is a function from $L$ to $k$, define the eigenspace $V_{\lambda}$ and weight space $V^{\lambda}$ by

$$
\begin{aligned}
V_{\lambda} & =\{v \in V \mid x v=\lambda(x) v \text { for all } x \in L\}, \\
V^{\lambda} & =\left\{v \in V \mid \text { for all } x \in L,[x-\lambda(x)]^{i} v=0 \text { for some } i=i(x)\right\} .
\end{aligned}
$$

It is clear that $V_{\lambda}$ is a submodule of $V$ and that if $V_{\lambda} \neq 0$, then $\lambda$ must be linear and in fact a character of $L$ (i.e., a homomorphism from $L$ to $k$; equivalently, $\lambda$ is linear and $\lambda([L, L])=0)$. In $[\mathbf{5}$, Theorem 42] it was asserted that, if $k$ has characteristic zero, then $V^{\lambda} \neq 0$ implies $\lambda$ is linear. However, the proof tacitly assumed $L$ solvable. In [7], the further questions were raised: if $V^{\lambda}$ is nonzero, must $\lambda$ be a character, and must $V_{\lambda}$ be nonzero? One may also ask whether $V^{\lambda}$ must be a submodule. These questions appear to have been answered completely only for $L$ nilpotent $[\mathbf{4}$, $\S I I .4]$. In this note we answer all three questions affirmatively for arbitrary $L$ when $k$ is of characteristic zero.

THEOREM. Let $k$ have characteristic zero. Suppose $V^{\lambda} \neq 0$. Then

(a) every semisimple subalgebra of $L$ acts trivially on $V^{\lambda}$,

(b) $\lambda$ is a character,

(c) $V^{\lambda}$ is a submodule of $V$,

(d) $V_{\lambda} \neq 0$.

PROOF. Note that unless $\lambda$ is known to be linear, there is no natural way to preserve the hypothesis under extension of scalars ( $\lambda$ might not extend!).

(a) We will use the fact that a semisimple Lie algebra of endomorphisms on a finite-dimensional vector space is spanned by its semisimple elements. One way to see this is to note first that it is true for the standard two-dimensional representation of the split three-dimensional algebra, since if $e$ and $f$ correspond to $\left[\begin{array}{ll}0 & 1 \\ 0 & 0\end{array}\right]$ and $\left[\begin{array}{ll}0 & 0 \\ 1 & 0\end{array}\right]$, then $e+f, e+4 f$, and $[e, f]$ act semisimply and span the algebra. Since every irreducible representation of this algebra occurs in the symmetric algebra of this representation, hence has the desired property, the assertion now follows from [4, Chapter III, Theorem 17].

Received by the editors July 5, 1984 and, in revised form, January 14, 1985.

1980 Mathematics Subject Classification. Primary 17B10.

Key words and phrases. Finite-dimensional Lie algebra, Lie algebra module, weight space.

${ }^{1}$ Research partially supported by NSF grant no. MCS-8301700. 
If $x$ acts semisimply, then each $v \in V^{\lambda}$ is an eigenvector for $x$. Thus if $L$ is semisimple, each $v \in V^{\lambda}$ is a common eigenvector for $L$, so $\lambda$ must be a character. Since $L=[L, L], \lambda=0$. Thus $L$ acts trivially on $V^{\lambda}$.

(b) Suppose first that $L$ is solvable. Let $K$ be the algebraic closure of $k, L_{K}=$ $L \otimes_{k} K, V_{K}=V \otimes_{k} K$. Then $L_{K}$ is solvable and acts in the natural way on $V_{K}$, so by Lie's Theorem [5, Theorem 2.6 or 3 , Corollary $4.1 \mathrm{~A}]$, there exist $L_{K}$-invariant subspaces

$$
(0)=W_{0} \subset W_{1} \subset \cdots \subset W_{n}=V_{K}
$$

with $\operatorname{dim}_{K} W_{i}=i$. Suppose $0 \neq v \in V^{\lambda}$. Let $i$ be greatest with $v \notin W_{i}$. Let $\bar{v}$ be the image of $v$ in $\bar{W}=W_{i+1} / W_{i}$. Since $\bar{W}$ is one-dimensional, $\bar{W}=(\bar{W})_{\mu}=(\bar{W})^{\mu}$ for some character $\mu$ of $L_{K}$. It follows that $\lambda=\left.\mu\right|_{L}$, hence is a character of $L$.

Now let $L$ be arbitrary. Let $L=S \oplus R$ be the Levi decomposition of $L$, where $S$ is semisimple and $R$ is the radical of $L[\mathbf{4}, \S I I I .9$ or 2 , Théorème 1.6.9]. By (a), $\left.\lambda\right|_{S}=0$. By the above, $\left.\lambda\right|_{R}$ is a character. Suppose $s \in S$. Then $H=k s \oplus R$ is a solvable Lie algebra, so $\left.\lambda\right|_{H}$ is a character. In particular, $\lambda(\alpha s+\beta r)=\alpha \lambda(s)+\beta \lambda(r)$ for all $r \in R$, all $\alpha, \beta \in k$. It follows that $\lambda$ is linear on $L$. Also since $\left.\lambda\right|_{H}$ is a character, $\lambda([s, R])=0$. Since $[L, L]=S+[S, R]+[R, R]$, this shows that $\lambda$ is a character.

(c) Let $\rho$ be the representation of $L$ corresponding to $V$. Since $\lambda$ is a character, $x \mapsto \rho(x)-\lambda(x)$ is easily seen to be a Lie homomorphism from $L$ to $\operatorname{End}_{k} V$. Replacing $L$ by its image under this homomorphism, we may assume without loss of generality that $\lambda=0$. The result now follows from $[\mathbf{1}$, Chapter $7, \S 1$, Exercise 5] (due to G. Seligman).

(d) Let notation be as in (b). Since $V^{\lambda}$ is an $R$ module, $R_{K}$ acts on $\left(V^{\lambda}\right)_{K}$ and therefore has, by Lie's Theorem, a common eigenvector $0 \neq v \in\left(V^{\lambda}\right)_{K}$. Considering $\left(V^{\lambda}\right)_{K}$ as an $R$ module, $\left(V^{\lambda}\right)_{K}=\left(\left(V^{\lambda}\right)_{K}\right)^{\nu}$ where $\nu=\left.\lambda\right|_{R}$. Thus $x v=\lambda(x) v$ for all $x \in R$. If $v=\sum v_{i} \otimes a_{i}$ where the $a_{i} \in K$ are linearly independent over $k$ and $v_{i} \in V^{\lambda}$, it follows, since $\lambda(x) \in k$, that $x v_{i}=\lambda(x) v_{i}$ for each $i$ and all $x \in R$. Since $S$ acts trivially on $v_{i}$, we thus have $v_{i} \in V_{\lambda}$.

Part (a) of the Theorem allows us to remove the restriction of solvability from Corollary 5 of $[\mathbf{7}]$. U( ) denotes the universal algebra functor.

COROLLARY. Let $L$ be a finite-dimensional Lie algebra over a field of characteristic zero. Let $B=\bigcap\left\{\operatorname{ker} \alpha \mid \alpha \in L^{*}\right.$ and $\left.L_{\alpha} \neq 0\right\}$. Then $\mathrm{U}(L)^{\lambda} \subseteq \mathrm{U}(B)$ for every $\lambda$.

PROOF. See [7, Corollary 4].

REMARK. G. Bergman has suggested, in connection with the proof of the Theorem, that the sum $I$ of all semisimple subalgebras of $L$ should be an ideal of $L$. This does not seem to appear anywhere in the literature, but for $k$ algebraically closed is an immediate consequence of a result of Krempa [6, Corollary 1], since $I$ is clearly invariant under all automorphisms of $L$. The following argument applies for any field of characteristic zero. Let $x \in N$, the nilpotent radical of $L$. For $s \in I$ and $\lambda \in k, I$ contains

$$
\exp (\operatorname{ad} \lambda x) s=s+\lambda(\operatorname{ad} x) s+\lambda^{2}(\operatorname{ad} x)^{2} s+\cdots+\lambda^{j}(\operatorname{ad} x)^{j} s,
$$

where $(\operatorname{ad} x)^{j+1}=0$. Since $k$ is infinite, a Vandermonde determinant argument shows that $(\operatorname{ad} x) s \in I$. Thus $[I, N] \subseteq I$. With $S, R$ as above, $R=R_{0}+R_{1}$ where 
$\left[S, R_{0}\right]=0$ and $R_{1}$ is the sum of nontrivial irreducible $S$ modules ( $S$ acting via ad). Then $R_{1}=\left[S, R_{1}\right] \subseteq N$, so $[S, R]=\left[S, R_{1}\right] \subseteq[S, N] \subseteq[I, N] \subseteq I$. Thus, $[S, L]=[S, S]+[S, R] \subseteq I$. Since $S$ may be chosen to contain any semisimple subalgebra of $L$, this shows that $I$ is an ideal.

ACKNOWLEDGEMENT. The author wishes to thank the referee of an earlier version of this paper for suggestions (especially the exercise from [1]) which greatly simplified the proof of the theorem.

\section{REFERENCES}

1. N. Bourbaki, Groupes et algèbres de Lie, Chapters 7 and 8, Publications Scientifiques et Industrielles 1364, Hermann, Paris.

2. J. Dixmier, Algèbres enveloppantes, Gauthier-Villars, Paris, 1974.

3. J. Humphreys, Introduction to Lie algebras and representation theory, Springer, New York, 1972.

4. N. Jacobson, Lie algebras, Wiley-Interscience, New York, 1962.

5. I. Kaplansky, Lie algebras and locally compact groups, Univ. of Chicago Press, Chicago, 1971.

6. J. Krempa, On invariant subspaces of locally finite derivations, Bull. London Math. Soc. 12 (1980), 374-376.

7. M. Smith, Automorphisms of enveloping algebras, Comm. Algebra 11 (1983), 1762-1802.

Department of Mathematics, University of TeXas, Austin, TeXas 73712 